\title{
A Fine Positronium Puzzle
}

\section{A high-precision measurement of positronium's fine structure delivers a puzzling discrepancy with predictions from quantum electrodynamics.}

\section{By Matteo Rini}

$\square$ ositronium is a hydrogen-like atom made of one electron and one positron (antielectron). Since positronium contains no protons or neutrons, nuclear interactions can be neglected, and the atom can be accurately described solely by quantum electrodynamics (QED)-the quantum counterpart of classical electromagnetism. Hence, positronium is an ideal system to test QED and to look for deviations that could indicate physics beyond the standard model. With this aim, David Cassidy and co-workers at University College London, UK, characterized the "fine structure" of positronium with unprecedented precision, revealing discrepancies with QED predictions [1].

An atom's fine structure describes the splitting of its energy levels due to electron spins and relativistic effects. Measuring it accurately for positronium is challenging, because the system annihilates within hundreds of nanoseconds of being produced. Reporting the first new fine-structure measurements in over 25 years, Cassidy and his colleagues detail the steps they took to improve accuracy and minimize systematic experimental errors. Specifically, they used lasers to selectively prepare positronium

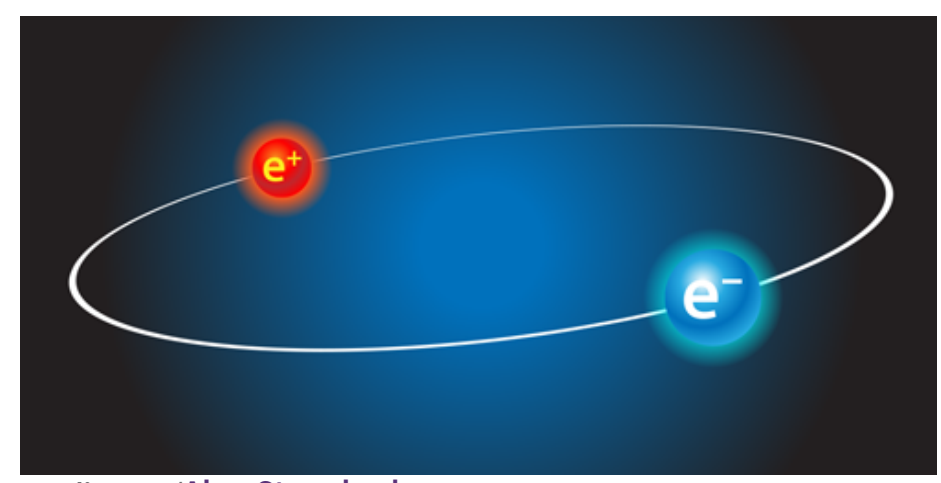

Credit: APS/Alan Stonebraker in an electronic state in which its lifetime was prolonged; they cooled the atoms to minimize Doppler effects that broaden the atomic lines; and they measured the transitions using low-power microwaves that don't significantly shift the atomic energy levels.

The team showed that the observed frequency of a specific positronium transition is about one part in a thousand larger than predicted by QED-a difference that significantly exceeds the estimated error bars. With further improvements, measurements of this transition and others may soon be able to explain this deviation and assess whether it implies new physics.

This research is published in Physical Review Letters.

Matteo Rini is the Deputy Editor of Physics.

\section{REFERENCES}

1. L. Gurung et al., "Precision microwave spectroscopy of the positronium $n=2$ fine structure," Phys. Rev. Lett. 125, 073002 (2020). 